\title{
The Neuroinflammatory Response Induced by PAF Can Be Attenuated by BN52021 Administration
}

\author{
Ruizhang Han ${ }^{1}$, Jinjia $\mathrm{Hu}^{2 *}$, Guojun Su${ }^{1}$ \\ ${ }^{1}$ Department of Neurosurgery of 98th Hospital of PLA, Huzhou, China; ${ }^{2}$ Medical College of Shanghai Jiaotong University, Shanghai, \\ China. \\ E-mail: ${ }^{*}$ jjhu2609@126.com
}

Received October $6^{\text {th }}, 2011$; revised November $17^{\text {th }}, 2011$; accepted November $29^{\text {th }}, 2011$.

\begin{abstract}
Objective: The purpose of this study was to determine whether BN52021, a platelet-activation factor receptor (PAFR) antagonist, could provide neuroprotection from the cytotoxic effects of PAF-induced neuroinflammation. Methods: The inflammagen platelet-activation factor (PAF) was first added to cultured PC12 cells. BN52021 was then added 24 hours later, survival rate and rate of apoptosis of the PC12 cells was determined by the MTT method and flow cytometry. In addition, PAF was injected into the fourth ventricle, and the effect of BN52021 administration was determined in rats. Results: PAF induced apoptosis in cultured PC12 cells, and BN52021 administration protected PC12 cells from $P A F$-induced apoptosis. When PAF is injected into the fourth ventricle, PAF induces acute neuroinflammation in the whole brain of rats. Acute PAF infusions also impaired spatial recognition in rats. The peripheral administration of BN52021 (i.p.) protected the rats from this impairment in spatial recognition. Conclusion: The PAFR antagonist $B N 52021$ provides neuroprotection from the cytotoxic effects induced by the inflamagen PAF.
\end{abstract}

Keywords: Neuroinflammation, Spatial Memory, Platelet-Activation Factor, BN52021

\section{Introduction}

Brain injury is a common disease that threatens human health [1]. Brain injury can be divided into types including primary and secondary [2]. Secondary brain injury or acute neuroinflammation occurs within hours or days after a brain injury, and this brain injury consists of neuron damage and nervous system dysfunction that is associated with specific biochemical processes. Learning and memory deficits represent common sequelae of secondary injury to the brain, and could seriously affect the quality of life of patients [3]. Various neuroinflammatory factors play an important role in secondary brain damage, including interleukin $1-\alpha$, interleukin $1-\beta$, prostaglandins, tumor necrosis factor, etc. PAF represents one of the strongest inflammatory factors in the central nervous system. Previous studies have found that a marked rise in PAF and prostaglandin levels were associated with brain inflammation [4]. Therefore, PAF is the key link in the regulation of neuroinflammation. The purpose of the current study was to investigate whether BN52021, PAFR antagonist, could provide neuroprotection from the cyto- toxic effects induced by PAF in vitro and in vivo.

\section{Materials and Methods}

\subsection{Cell Culture}

PC12 cells were cultured in MEM/F-12 (1:1) medium (Invitrogen, USA) supplemented with $10 \%$ fetal calf serum (FCS, Invitrogen), $100 \mathrm{U} / \mathrm{ml}$ penicillin, and 100 $\mu \mathrm{g} / \mathrm{ml}$ streptomycin. The PC12 cells were cultured in a humidifed atmosphere containing $5 \% \quad \mathrm{CO}_{2}$ at $37^{\circ} \mathrm{C}$. When the cells reached the proper confluence, they were placed into a serum-free medium. The cells were incubated with PAF $(0.1,0.3,0.6 \mu \mathrm{mol} / \mathrm{L})$ for 24 hours or incubated with PAF $(0.3 \mu \mathrm{mol} / \mathrm{L})$ for three hours. Without changing the culture medium, the cells were then treated with BN52021 (Long He Co.; low dose group: 1 $\mathrm{mg} / \mathrm{L}$; high dose group: $5 \mathrm{mg} / \mathrm{L}$ ) for an additional 48 hours.

\subsection{MTT Assay of Cell Viability}

PC12 cells were plated at $5 \times 10^{4}$ cells/well $(200 \mu \mathrm{l})$ into 96 well plates, and allowed to adhere and grow until they 
reached the required confluence. After drug treatment, the survival of cells was determined by MTT assay. Briefy, after incubation with $20 \mu \mathrm{l}$ MTT $(5 \mathrm{mg} / \mathrm{ml}$, Sigma) at $37^{\circ} \mathrm{C}$ for 3 hours, living cells containing MTT formazon crystals were made soluble in $200 \mu$ dimethylsulfoxide (DMSO, Sigma). The absorbance of each well was measured using a microculture plate reader with a test wave length of $570 \mathrm{~nm}$ and a reference wave length of $655 \mathrm{~nm}$. The light absorption value was measured to determine formazan absorption.

\subsection{Measurement of Apoptosis by Flow Cytometry}

To determine whether PAF-triggered cell death was apoptotic, we measured cell apoptosis using an AnnexinVFITC Apoptosis Detection Kit (BD Pharmingen, USA) according to the manufacturer's instructions. Briefy, the cells were plated at a density of $5 \times 10^{5}$ cells $/ \mathrm{ml}$ into six well plates, and cultured until the cells reached $70 \%$ confluence. After drug treatment, cells were trypsinized, collected by centrifugation. The cells were then resuspended in $200 \mu \mathrm{l}$ of binding buffer (provided by the manufacturer). This buffer contained $5 \mu \mathrm{l}$ of annexin V-FITC and propidium iodide (PI). The cells were incubated for 15 minutes at room temperature. All samples were kept on ice and analyzed immediately with a FACSCalibur flow cytometer (BD, USA).

\subsection{Animal Preparation}

Forty Sprague-Dawley rats (approximately 4 months old and weighing $200 \mathrm{~g}$ ) were divided into four groups, including a control group, a model group, a low dose group and a high dose group. The rats were anesthetized with $20 \%$ urethane $(1300 \mathrm{mg} / \mathrm{kg}$, i.p). The rats first were placed in a stereotaxic instrument with the incisor bar set $3 \mathrm{~mm}$ below the earbars (i.e., plat skull), and the scalp was incised and retracted. A hole was drilled in appropriate location in the skull with a dental drill. PAF $(0.3$ $\mu \mathrm{g} / \mathrm{kg}$, dissolved in artificial CSF, $5 \mu \mathrm{g} / \mu \mathrm{l})$ was injected into the fourth ventricle. Eight control rats were injected with artificial CSF. The next day after operation, the low dose and high dose group rats were treated with BN52021. BN52021 was first dissolved in DMSO, and then PBS was added at the following concentrations (i.p.): 0.01 $\mathrm{mol} / \mathrm{L}$ PBS, $1 \mathrm{mg} / \mathrm{kg}$ PBS (low dose), and $5 \mathrm{mg} / \mathrm{kg}$ PBS (high dose).

\subsection{Behavioral Testing}

Behavioral testing began 6 days after injection (i.p.). The Morris water maze test was used to measure the rats' ability to learn and remember spatial cues. The navigational test was conducted in a dark airtight room. The pool was divided into four quadrants, and a platform was placed in one of the quadrants. The rats were randomly placed in the water from four different directions. The rats were then allowed 90 seconds to find the platform. If the rats failed to find the platform within 90 seconds, they were placed on the platform where they stayed for 30 seconds. This test was conducted once per day for 4 days. The platform was then removed, and the rats were placed in the center of the pool to find the platform in order to test their special memory. A computer recorded (1) the number of times that the rat passed the platform and (2) the percentage of swimming distance in the platform quadrant to the total distance.

\section{Results}

\subsection{The Cytotoxicity of PAF}

MTT assay of cell viability showed that the absorbance value decreased gradually with the increase of PAF concentration. The absorbance value of 4 PAF concentration groups was shown in Figure 1. Measurement of Apoptosis by Flow Cytometry was shown in Figure 2: As the concentration of PAF increased, the percentage of apopotosis of cells increased.

\subsection{The Protection of BN52021 from PAF Cytotoxiciy}

MTT Assay of Cell Viability to test PAF cytotoxicity was shown in Figure 3. Both low dose and high dose of BN52021 could effectively increase light absorption of A value. Measurement of Apoptosis by Flow Cytometry was shown is Figure 4. Compared with PAF group, both low dose and high dose of BN52021 could decrease the percentage of apopotosis of cells.

\subsection{Morris Water Test of Spatial Memory}

The rats appeared normal and did not lose weight following the PAF injection. The training results of the four tests in place navigation test (i.e., evasive latency) are shown in Figure 5. The percentage of swimming distance in the platform quadrant relative to the total dis-

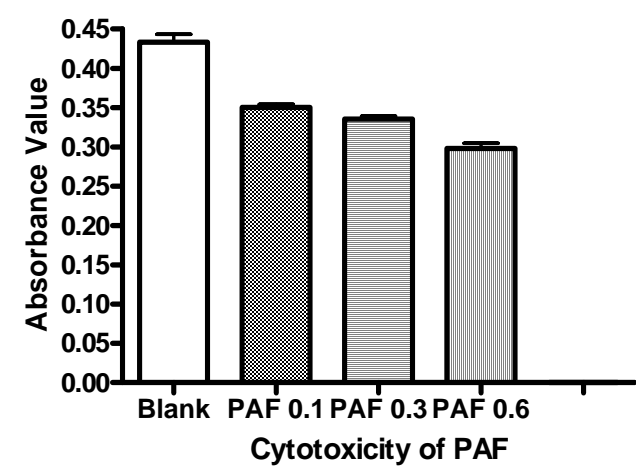

Figure 1. MTT Assay of cell viability. 

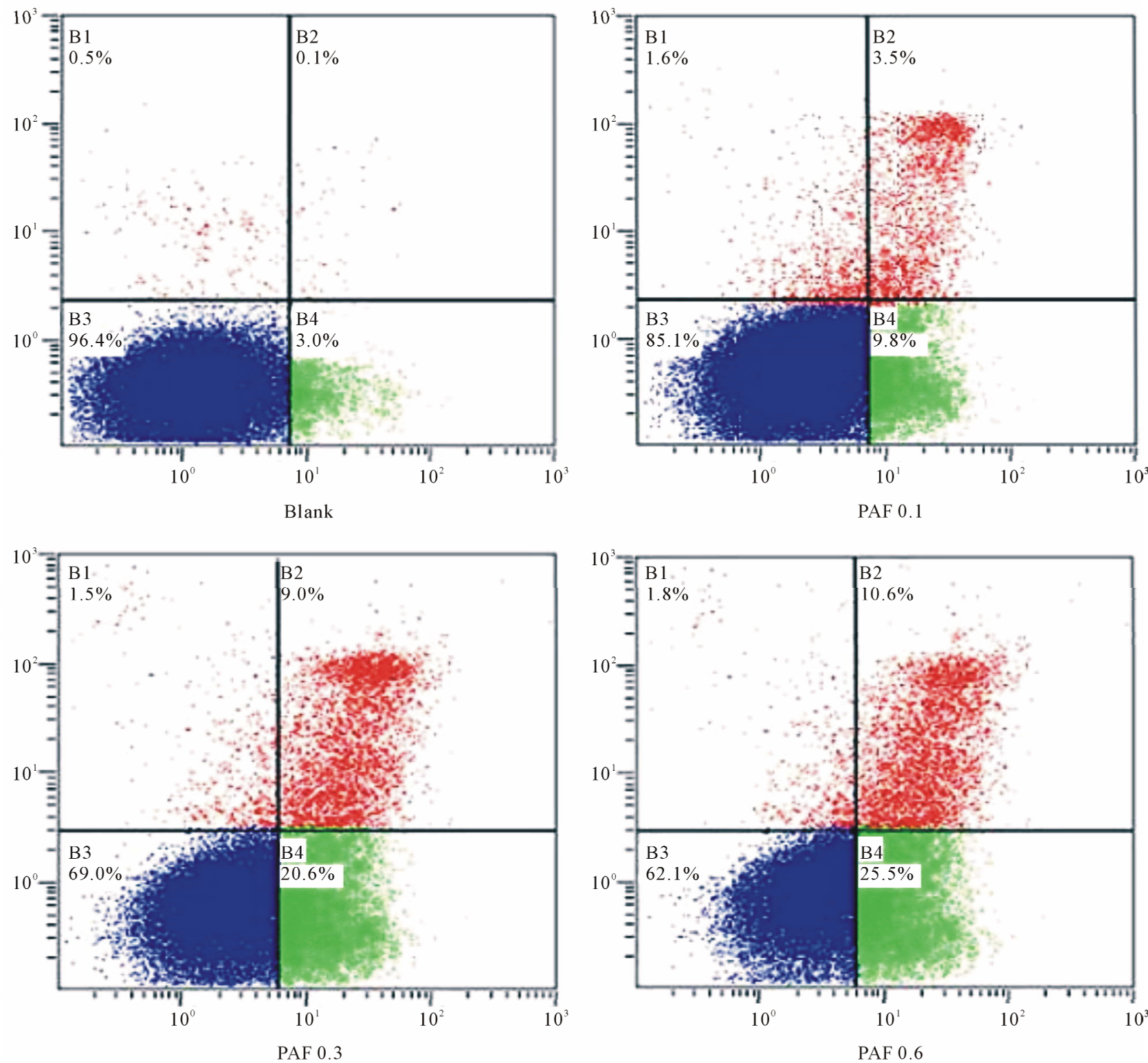

Figure 2. Measurement of apoptosis by flow cytometry.

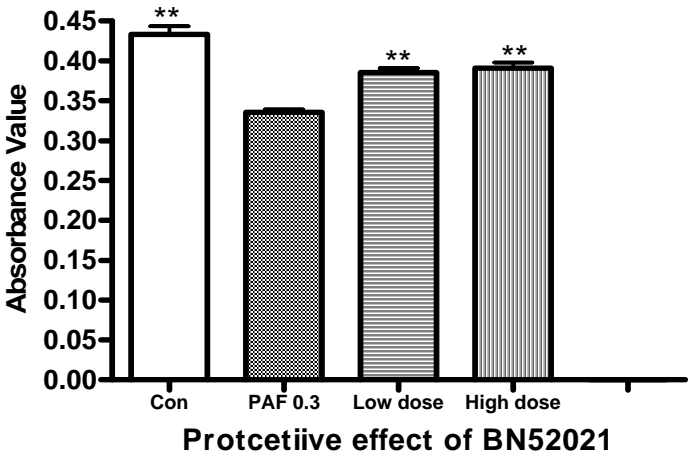

Figure 3. MTT Assay of cell viability: $* * \mathbf{P}<0.01$, compared with PAF 0.3 group. tance and the number of times the rats passed the platform are shown in Figure 6. The control group and the two treatment groups showed significant increases in both the number of times passing the platform and the percentage of the swimming distance, as compared to the model group.

\section{Discussions}

The primary mechanism associated with secondary brain injury is neuroinflammation and cerebral edema. Therefore, the inhibition of neuroinflammation likely represents a crucial therapy for the treatment of secondary brain injury $[5,6]$. As one of the strongest inflammatory factors, 

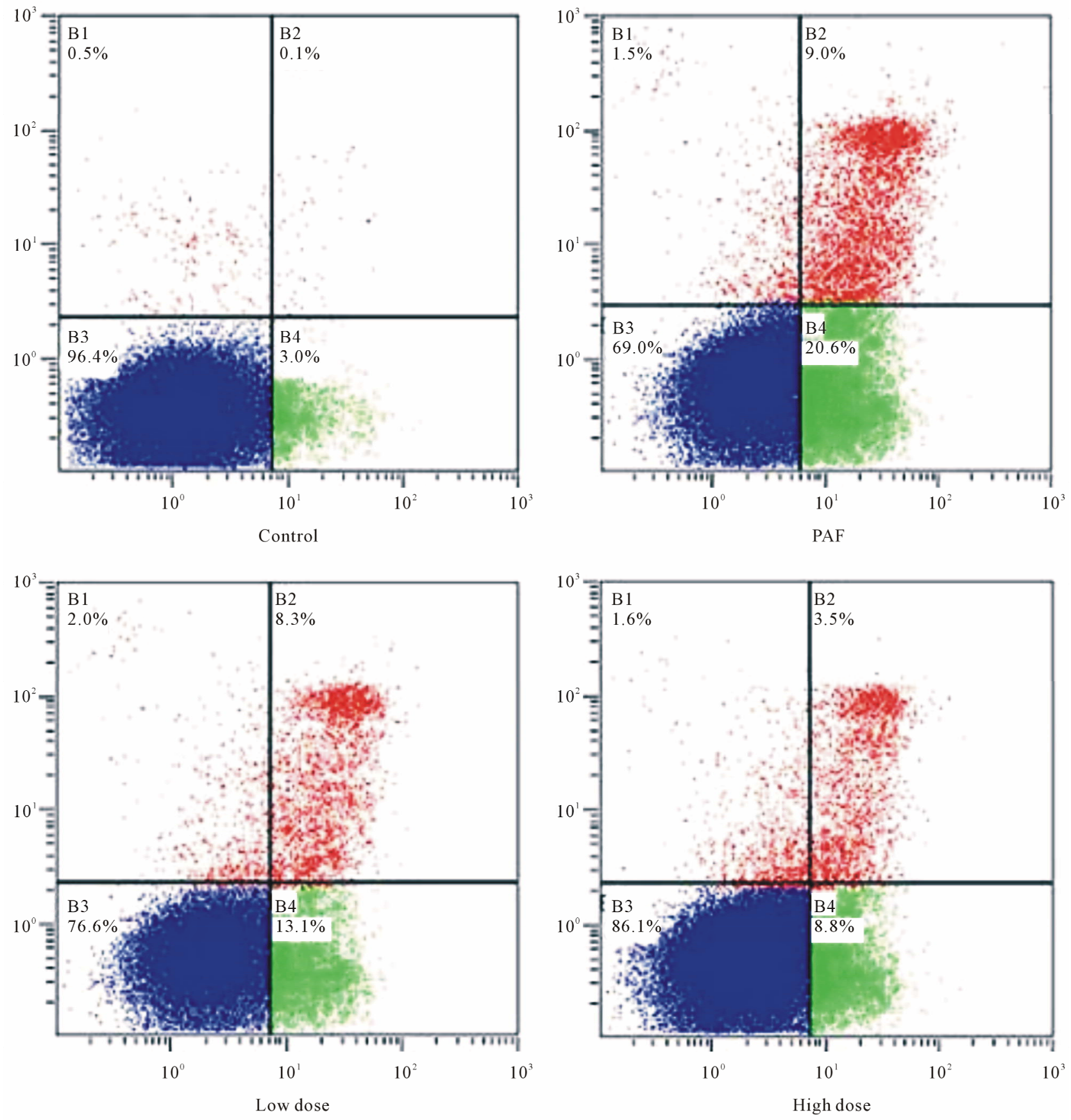

Figure 4. Measurement of apoptosis by flow cytometry.

PAF plays a key role in the modulation of neuroinflammation [7]. PAF is expressed in neurons as well as neuroglial cells, and previous studies report that PAF is increased after brain injury [8]. Therefore, a high dose of PAF could affect neurotoxicity [9]. Likewise, the PAFR antagonist-E5880 could protect neurons and neuroglial cells from neurotoxicity induced by glutamine [10].

In present study, PAF induced apoptosis and cell death when added to cultured PC12 cells. As the concentration of PAF increased, cell death increased in a similar manner. However, cell death markedly decreased when BN52021, a PAF antagonist, was administered prior to PAF. These findings demonstrate that BN52021 can protect PAF-induced cytotoxicity in vitro. Additionally, the central inflammatory response produced by the infusion of PAF produced a significant deficit in spatial memory, 


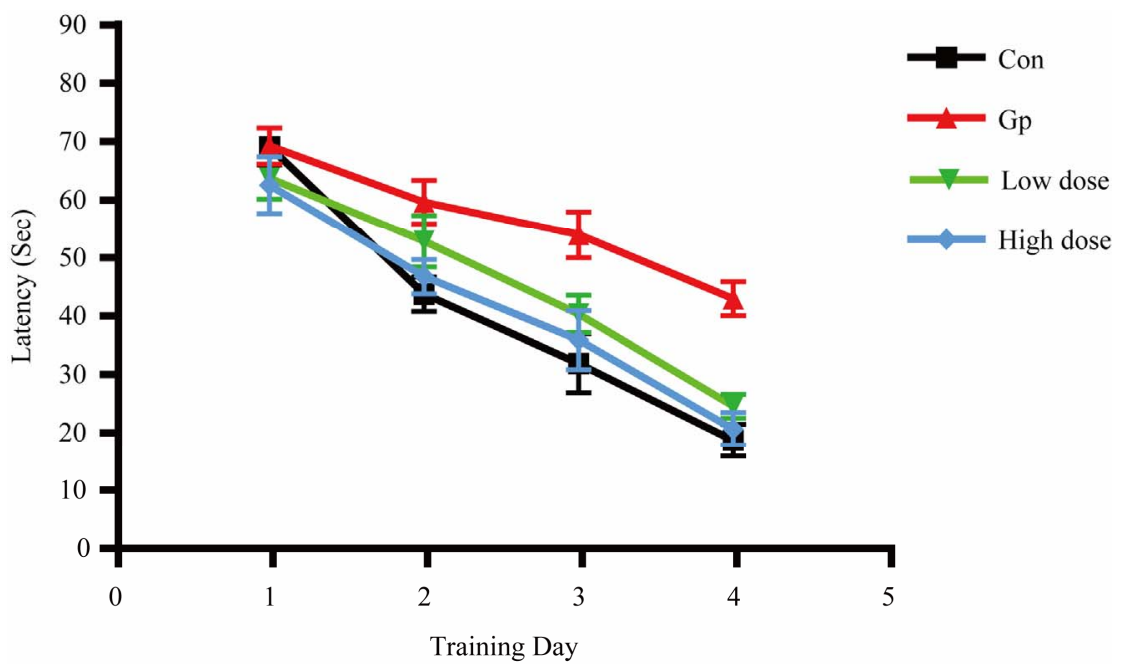

Figure 5. The average escape latency of rats in 4 tests in each group. With the increase of training time, evasive latency was shortened in all the 4 groups, and the slope of evasive latency in PAF group was the least. The evasive latency of day 3 or day 4 in PAF group is significant compared with other 3 groups $(P<0.05$ or $P<0.01)$. Moreover, 2 treatment groups showed no significant difference compared with the control group. $* \mathbf{P}<0.01$ vs $\mathbf{P A F}$ group.

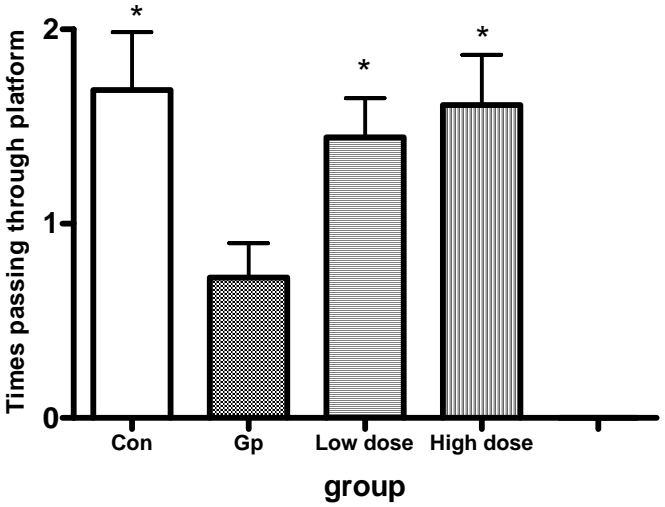

(a)

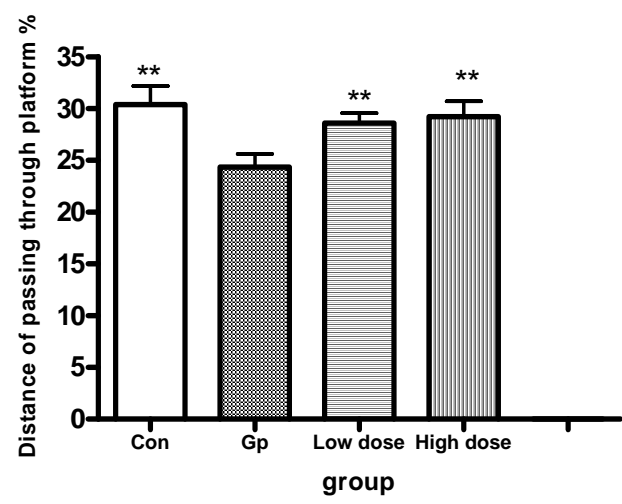

(b)

Figure 6. Results of the spatial probe test. (a) The number of times that rats passed the platform. (b) The percentage of the swimming distance in platform quadrant to the total distance. The control group and the 2 treatment groups were all significantly different from PAF group in both the number of times passing the platform and the percentage of the swimming distance. None of the treatment groups showed any significant difference with control group. $* \mathbf{P}<0.05, * * \mathbf{P}<0.01$ vs model group.

as measured by the Morris water maze. Interestingly, this memory deficit was completely attenuated by the coadministration of BN52021. These results indicate that a PAFR antagonist protects neurons and neuroglial cells from neuroinflammation as well as spatial memory deficits. Therefore, PAFR antagonists may be a relevant compound for the treatment of brain injury, and BN52021 would be important to assess in clinical trials for brain injury.

\section{REFERENCES}

[1] X. D. Qu, R. Shrestha and M. D. Wang, "Risk Factors Analysis on Traumatic Brain Injury Prognosis," Chinese
Medical Sciences Journal, Vol. 26, No. 2, 2011, pp. 98102. doi:10.1016/S1001-9294(11)60027-8

[2] J. M. Simard, Z. Geng, S. K. Woo, S. Ivanova, et al., "Glibenclamide Reduces Inflammation, Vasogenic Edema, and Caspase-3 Activation after Subarachnoid Hemorrhage," Journal of Cerebral Blood Flow \& Metabolism, Vol. 29, No. 2, 2009, pp. 317-30. doi: $10.1038 / \mathrm{jcbfm} .2008 .120$

[3] R. L. Wood and N. A. Rutterford, "Demographic and Cognitive Predictors of Long-Term Psychosocial Outcome Following Traumatic Brain Injury," Journal of the International Neuropsychological Society, Vol. 12, No. 3, 2006, pp. 350-358. doi:10.1017/S1355617706060498

[4] W. L. Titsworth, N. K. Liu and X. M. Xu, "Role of Sec- 
retory Phospholipase a(2) in CNS Inflammation: Implications in Traumatic Spinal Cord Injury," CNS Neurological Disorders-Drug Targets, Vol. 7, No. 3, 2008, pp. 254-269. doi:10.2174/187152708784936671

[5] X. Zhou, J. Zhou, X. Li, C. Guo, et al., "GSK-3 $\beta$ Inhibitors Suppressed Neuroinflammation in Rat Cortex by Activating Autophagy in Ischemic Brain Injury," Biochemical and Biophysical Research Communications, Vol. 411, No. 2, 2011, pp. 271-275.

doi:10.1016/j.bbrc.2011.06.117

[6] S. S. Dai and Y. G. Zhou, "Adenosine 2A Receptor: A Crucial Neuromodulator with Bidirectional Effect in Neuroinflammation and Brain Injury," Review in the Neuroscience, Vol. 22, No. 2, 2011, pp. 231-239. doi:10.1515/RNS.2011.020

[7] Y. Deng, W. Fang, Y. Li, et al., "Blood-Brain Barrier Breakdown by PAF and Protection by XQ-1H Due to
Antagonism of PAF Effects," European Journal of Pharmacology, Vol. 616, No. 1-3, 2009, pp. 43-47. doi:10.1016/j.ejphar.2009.06.017

[8] T. Tokutomi, H. Maruiwa, M. Hirohata, et al., "Production of Platelet-Activating Factor by Neuronal Cells in the Rat Brain with Cold Injury," Neurological Research, Vol. 23, No. 6, 2001, pp. 605-611. doi: $10.1179 / 016164101101199081$

[9] H. S. Lustig, J. Chan and D. A. Greenberg, "Comparative Neurotoxic Potential of Glutamate, Endothelins, and Platelet-Activating Factor in Cerebral Cortical Cultures," Neuroscience Letters, Vol. 139, No. 1, 1992, pp. 15-18. doi:10.1016/0304-3940(92)90847-Z

[10] S. Ishii and T. Shimizu, "Platelet-Activating Factor (PAF) Receptor and Genetically Engineered PAF Receptor Mutant Mice," Progress in Lipid Research, Vol. 391, 2000, pp. 41-48. doi:10.1016/S0163-7827(99)00016-8 